\title{
siRNA-mediated mesothelin silencing for treatment of mesothelioma
}

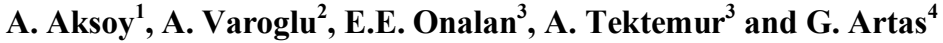 \\ ${ }^{1}$ Department of Medical Oncology, Medical Faculty, Firat University, \\ Elazig, Turkey \\ ${ }^{2}$ Department of Neurology, Medical Faculty, Medeniyet University, \\ Istanbul, Turkey \\ ${ }^{3}$ Department of Medical Biology and Genetics, Elazig, Turkey \\ ${ }^{4}$ Department of Pathology, Medical Faculty, Firat University, Elazig, Turkey
}

Corresponding author: A. Aksoy

E-mail: aaksoy@firat.edu.tr

Genet. Mol. Res. 21 (1): gmr18955

Received September 08, 2021

Accepted September 21, 2021

Published January 24, 2022

DOI http://dx.doi.org/10.4238/gmr18955

\begin{abstract}
It is known that mesothelin (MSLN) is overexpressed in some cancers, and that it plays a role in cell growth through Wnt family member 1 protein. Malignant transformation usually occurs with disruption of autoregulation of autophagy-related genes. We examined the effect of MSLN on survival and clinicopathological features in mesothelioma cases, as well as variations in genes associated with autophagy, invasion, apoptosis-related genes after siRNA-mediated MSLN silencing transfection in a mesothelioma cell line (SPC212). MSLN expression was analyzed, immunohistochemically, in formalinfixed paraffin-embedded 60 mesothelioma cases. MSLN expression was categorized by median MSLN histoscores as low (L), high (H). The correlation between the levels of MSLN expression, clinicopathological features, and survival was determined in mesothelioma cases. The siRNA-mediated MSLN incubated SPC212 cells were transfected and compared to negative control siRNAs. mRNA levels were determined for autophagy, invasion, and apoptosis related-genes with RT-PCR, and western blotting in SPC212 cells after MSLN silencing. All of the cases were immunoreactive for MSLN expression. H-MSLN was associated with a favorable prognosis according to Kaplan-Meier survival analysis, but Cox regression analysis revealed that only stage was a significant independent factor for estimating survival. MSLN overexpression was
\end{abstract}


significantly higher in early-stage, mesothelioma cases without nodal involvement. Significant silencing in MSLN was found (87.5\%) after siRNA applications. Apoptosis and autophagy were upregulated by increasing apoptosis-related genes, BAK1, BAX, CASP1-7, and autophagy-related genes, ATG2, ATG16L1. Cell proliferation was knocked down predominantly by inhibiting the invasion-related genes, MMP1, 10, 11, 13, in SPC212 cell lines. MSLN silencing was determined to significantly increase CASP 3 , but did not change LC3 in western blotting, respectively. In conclusion, siRNA-mediated silencing MSLN can promote apoptosis, autophagy, and also partially inhibit proliferation. We suggest that MSLN can serve as a potential therapeutic target in mesothelioma.

Key words: Autophagy; Apoptosis; Mesothelioma; Mesothelin; Proliferation; Survival

\section{INTRODUCTION}

Mesothelioma is a rare mesothelial cell tumor, located in pleural, pericardial, and peritoneal cavities. Furthermore, it has a poor prognosis and is common in specific areas with high soil asbestos content. Personalized medicine has emerged with the understanding of the molecular mechanisms of cancer. Recent studies demonstrated that immune checkpoint inhibitors, nivolumab, and ipilumumab, have an advantage as first-line therapy for mesothelioma (Van Zandwijk et al., 2020; Baas et al., 2021). Mesothelioma is resistant to chemotherapy, and a higher number of relapses are observed due to resistance to apoptosis (NCCN, 2021).

After long-term inflammation, asbestos fibers activate the AP/TNF alpha/ nuclear factor kappa-light-chain-enhancer of activated B cells (Nf-kB) autocrine pathway, leading to DNA damage and uncontrolled cell growth (Kim et al., 2006; NCCN, 2021). These abnormal cells, exposed to asbestos for a long time, try to adapt themselves to their microenvironment. When this function, which allows the cell to adapt to challenging conditions, is disrupted, a malignant transformation is observed (Gozuacik et al., 2004). Malign transformation often occurs with disruption of autoregulation in autophagy mechanisms. Autophagy and energy metabolisms are strongly associated. MSLN expression entails surface cell glycoproteins expression in mesothelial cells. Certain cancers such as mesothelioma, gastric, pancreatic, ovarian, and triple-negative breast cancers are associated with high titers of MSLN (Chang et al., 1996; Bharadwaj et al., 2011; Chang et al., 2012; Del Bano et al., 2019). Although the biological functions of MSLN are still not fully understood, it is known that MSLN activates and regulates matrix metalloproteinases (MMPs) and neurokinin B (NKb). Some cancers with MSLN overexpression activate Wnt family member 1 (Wnt-1) proteins during maturation, differentiation, and apoptotic stages. Therefore, MSLN is considered an excellent candidate for targeted therapy in cancers with MSLN overexpression (Rump et al., 2004; Bharadwaj et al., 2011; Yeo et al., 2021).

Studies demonstrated that miRNAs targeted in the treatment of several cancers could either be silenced or imitated. In previous studies, certain miRNAs were employed as new potential targets in cancer treatment in recent years (Fukumoto et al., 2015). 
Furthermore, recent studies demonstrated that the c-Jun N-terminal kinase (JNK) pathway plays a potential role in the physiopathology of peritoneal mesothelioma (Srivastava et al., 2015). The detection of the molecular changes in the etiopathogenesis and the invasion of mesothelioma would determine the essential goals of treatment. In vitro and in vivo studies on blocking of MSLN in mesothelioma have not been reported. In our study, we investigated the results of siRNA-mediated MSLN silencing in mesothelioma cell lines in autophagy, invasion, and apoptosis-related gene panels, and whether MSLN was a prognostic biological marker.

\section{MATERIAL AND METHODS}

\section{Case selection}

MSLN expression was analyzed in biopsy material obtained from 60 mesothelioma cases. Before the study, Firat University Ethics Committee approval was achieved to access the retrospective medical data of patients who were treated between 2014 and 2018 in Firat University, Medical Oncology Department. Participants signed informed consent forms.

\section{Tissue samples and immune histochemistry}

5- $\mu$ m-thick paraffin blocks of the tissues fixed with routine formalin buffer were transferred to poly-lysine slides. After obtaining sections from paraffin blocks, they were placed in an automated staining machine (Ventana Medical System. SN: 712299, REF: 750700, Arizona, USA) for MSLN (G1) (Mouse monoclonal $200 \mu \mathrm{m} / \mathrm{mL}, 1 / 200$, Santa Cruz). The preparations were inspected, assessed, and photographed with a Leica DM500 microscope.

The histoscores were determined based on the intensity $(0.1:<25 \%, 0.4: 26-50 \%$, $0.6: 51-75 \%, 0.9: 76-100 \%)$ and severity of immune reactivity $(0:$ no, 0.5 positive: very little, 1 positive: little, 2 positive: medium, 3 positive: severe) (Figure 1a-b). The histoscores were calculated with the "intensity X severity" formula (Carter et al. 2004; Alì et al., 2013; Yalçin et al., 2019). The MSLN histoscores were categorized into two subgroups: those that were lower and higher than median values $(\geq 2.7$ high MSLN group, $<2.7$ low MSLN group.

\section{Cell culture}

RPMI-1640 medium (Cat. No. R0883, Sigma-Aldrich, Germany) with fetal bovine serum 10\%, (Cat. No. F6178, Sigma-Aldrich, the USA) was implemented to culture SPC212 cells (ATCC ${ }^{\circledR}$ CRL-1435 ${ }^{\mathrm{TM}}$ ). SPC212 cells were produced in incubators with $5 \% \mathrm{CO}_{2}$ and $95 \%$ air at $37^{\circ}$ (Nuve, Turkey)

\section{siRNA transfection}

Based on the producer's instructions, SPC212 cells were emplacement on 6well cell culture plates, bred in containers with HiPerfect ${ }^{\circledR}$ transfection test subject (Cat. No. 301704, Qiagen, Germany) after one day. They were transfected with MSLN- 
specific siRNA (Cat. No. GS10232, Qiagen, the USA) or negative control siRNA (Cat. No. 1027280, Qiagen, the USA). We used human gene tests (Cat. No. PPH19412A, Qiagen, Germany) on MSLN with quantitative Real Time-Polymerase Chain Reaction (qRT-PCR) to observe the presence of knockdown. The percentage of siRNA silencing with the $\Delta \triangle \mathrm{CT}$ Mean $=\Delta \mathrm{CT}$ MSLN-siRNA $-\Delta \mathrm{CT}$ Negative Control; Fold Change $=2^{-}$ $\Delta \Delta \mathrm{CT}$; Percentage of Silence $=100 \times(1-$ Fold Change $)$ formula calculated qRT-PCR results.

\section{Total RNA isolation in cell culture}

Based on the producer's instructions, RNA was isolated from SPC212 cells with the Gene Jet RNA Purification kit (Cat. No. K0731, Thermo Scientific, Lithuania). The RNA pellet was resuspended out in 10-30 $\mu$ l nuclease-free water.

\section{Complementary DNA synthesis}

Based on the manufacturer's instructions, the PCR was conducted for complementary DNA (cDNA) synthesis with a High-Capacity cDNA Reverse Transcription Kit (Cat. No. 4368814, Applied Biosystems, the USA) and a thermal cycler device (Veriti, Applied Biosystems, Singapore) at $25^{\circ} \mathrm{C}$ for $10 \mathrm{~min}, 37^{\circ} \mathrm{C}$ for 120 $\min$, and $85^{\circ} \mathrm{C}$ for $5 \mathrm{~min}$.

\section{qRT-PCR Analysis}

The mRNA level gene expression tests employed by SYBR green-based autophagy panel (Cat. No. HATPL-I, Human Autophagy Primer Library, RealTimePrimers.com), apoptosis panel (Cat. No. HPA-I, Human Apoptosis Primer Library, RealTimePrimers.com), invasion panel (Cat. No. HTIM-I, Human Invasion Primer Library, RealTimePrimers.com). Tables 2 and 3 are summarized. These genes and their properties were determined in qRT-PCR Analysis on SPC212 cells and paraffin-embedded MSM tissues. The iTaq universal SYBR green supermix (Cat. No. 172-5121, Bio-Rad, and the USA) was prepared the mixture with for expressions of autophagy-apoptosis-related genes. The qRT-PCR system (7500 RT-PCR, Applied Biosystems, and Singapore) was employed to determine the mRNA expression degrees of the genes. To control the group in our study, we used Glyceraldehyde 3-phosphate dehydrogenase (GAPDH) (Cat. No. QT00079247, Qiagen, the USA). We used to determine the gene expression differences after the qRTPCR analysis the $2^{-\Delta \Delta C T}$ method. We repeated PCR measurements three times, and the reported measurements were based on this triplicate analysis.

\section{Western blotting}

We performed homogenisation of the cells via ice 1:10 (w/v) in a buffer containing $50 \mathrm{mM}$ Tris $(\mathrm{pH}$ 7.4), $0.1 \mathrm{mM} \mathrm{NaCl}, 1 \%$ Triton $\mathrm{X}-100,5 \mathrm{mM}$ EDTA, $1.0 \mathrm{mM}$ phenylmethylsulfonyl fluoride, $10 \mathrm{mg} / \mathrm{mL}$ aprotinin and $10 \mathrm{mg} / \mathrm{mL}$ leupeptin. We measured protein concentration for each sample according to the Lowry procedure using a protein assay kit (Sigma, St. Louis, MO, USA), and western blotting. We transferred separated 
proteins with SDS polyacrylamide gel electrophoresis to nitrocellulose membranes (Santa Cruz Biotechnology, Inc., Texas, and the USA). We used nitrocellulose blots blocked with $5 \%$ dry milk and probed with CASP3 (ab13847, Abcam, Cambridge, UK) and Lc-3 (sc398822, Santa cruse) primary antibodies at a dilution of 1:500. It was washed the blots and bred for $1 \mathrm{~h}$ with a secondary antibody, anti-mouse or anti-rabbit Ig peroxidase-conjugated (Santa Cruz Biotechnology, Inc., Texas, and the USA) at a dilution of 1:500. Chemi-glow detection reagents using chemiluminescent detection showed specific binding. We quantified scanning blots using a computerized software program (LabWorks 4.0; UVP, Inc., Cambridge, UK) the relative amount of immunoreactive bands on Western blots in arbitrary units. Primary human anti-beta actin and secondary anti-mouse antibodies were used to monitor beta-actin expression as a loading control.

\section{Statistical Analysis}

We used the statistical package for social sciences (IBM corp. Armonk, NY, the USA) version 22 for statistical analyses. To compare the clinicopathological characteristics among high-low MSLN expression groups, the Student's t-test was performed. To predict prognostic factors of overall survival (OS) Kaplan-Meier model, Cox proportional hazards regression model were performed. Spearmen/Pearson correlation was used to determining the parameters affecting the median overall survival (OS). We analyzed the qRT-PCR data with the $\Delta \Delta \mathrm{Ct}$ module at the Qiagen Gene Globe Data Analysis Center portal (http://www.qiagen.com/us/shop/genesand-pathways/data-analysis-center-overviewpage/).

The qRT-PCR module transformed the threshold cycle $(\mathrm{Ct})$ values to determine gene expressions. The outcomes in the $95 \%$ confidence interval (CI) were noted statistically significant $(\mathrm{P}<0.05)$.

\section{RESULTS}

\section{Tissue immune histochemistry results}

MSLN expression was determined in all cases. We categorized the histoscores of MSLN into two subgroups as values lower and higher than median values $(\geq 2.7$ high MSLN group, $<2.7$ low MSLN group). While in $66.7 \%$ of the cases MSLN expression was high, MSLN expression was low in 33.3\% of the cases (Figure 1a-b).

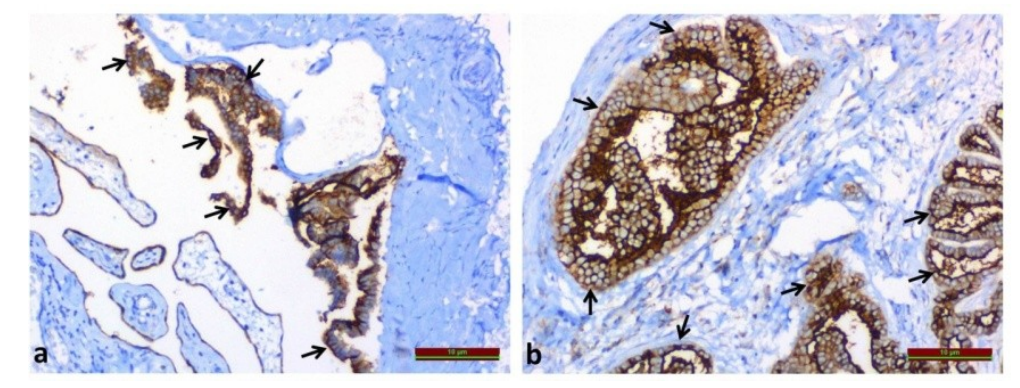

Figure 1. Histopathological evaluation for mesothelin (MSLN) (Haematoxylin \& Eosin (HE) X100), Immunoperoxidase X 100). 1a. Low (L)- MSLN expression, 1b. High (H)-MSLN expression. 
The general clinical characteristics of the cases and their relationship to MSLN expression are shown in Table 1.

Table 1. Association of mesothelin (MSLN) expression with demographic and clinicopathological parameters.

\begin{tabular}{|c|c|c|c|c|}
\hline & & Mesot & core & \\
\hline Variables & $n=60,(\%)$ & Low (n: 20) & High (n:40) & P-value ${ }^{\#}$ \\
\hline Gender & & & & 0.124 \\
\hline Woman & $20(33)$ & 4 & 16 & \\
\hline Man & $40(67)$ & 16 & 24 & \\
\hline Age $^{*}$ & $54.5 \pm 7.28^{*}$ & & & 0.014 \\
\hline$\leq 65 y$ & $50(83)$ & 20 & 30 & \\
\hline$>65 y$ & $10(17)$ & - & 10 & \\
\hline Mesothelioma & & & & 0.715 \\
\hline Pl-mesothelioma & $34(57)$ & 12 & 22 & \\
\hline Pr-mesothelioma & $26(43)$ & 8 & 18 & \\
\hline TNM-stage ${ }^{*}$ & & & & 0.007 \\
\hline I-II & $20(33)$ & 2 & 18 & \\
\hline III-IV & $40(67)$ & 18 & 22 & \\
\hline Lymph node metastases & & & & 0.002 \\
\hline No & $32(53)$ & 5 & 27 & \\
\hline $\mathrm{N} 1-2$ & $28(47)$ & 15 & 13 & \\
\hline CT status & & & & 0.392 \\
\hline $\mathrm{CT}(+)$ & $14(23)$ & 6 & 8 & \\
\hline C T(-) & $46(77)$ & 14 & 32 & \\
\hline RT status & & & & 0.669 \\
\hline RT (+) & $46(77)$ & 16 & 30 & \\
\hline RT (-) & $14(23)$ & 4 & 10 & \\
\hline
\end{tabular}

*: Mean \pm SD, ${ }^{*}$ : According to NCCN Guideline, AJJC-8 CT $^{3}$ : Chemotherapy, RT: Radiotherapy, MSM: Mesothelioma, MSLN: Mesothelin, Pr: Peritoneal, Pl: Pleural, (+): Received, (-): Not-received, \#Mann -Whitney U test used, TNM: T: tumor size, N: Nodal status, M: Metastases, $¥$ : According to AJCC 8 (Edge et al.,2017)

MSLN expression was also strongly correlated with age, stage, nodal involvement. MSLN overexpression was significantly higher in mesothelioma cases with younger, early-stage, and without nodal involvement $(\mathrm{P}=0.014, \mathrm{P}=0.007$, and $\mathrm{P}$ $=0.002$, respectively) as shown in Table 1 .

\section{Survival evaluation}

The median follow-up period of the cases was 24 months (range; 3 - 92).

The median overall survival was 35 months $(95 \% \mathrm{CI}$ : $27.33-42.66)$ in the $\mathrm{H}-$ MSLN group, and it was 11 months (95\% CI: 3.11-18.89) in the L- MSLN group (P = 0.001) (Figure 2).

Although the woman gender, overexpression of MSLN, early-stage, cases without nodal involvement were statistically favorable prognostic factors for survival according to Kaplan Meier analysis, Cox Regression analysis revealed that only stage of mesothelioma cases an independent prognostic factor for survival $(\mathrm{P}<0.05)$, as shown in Tables 2 . 

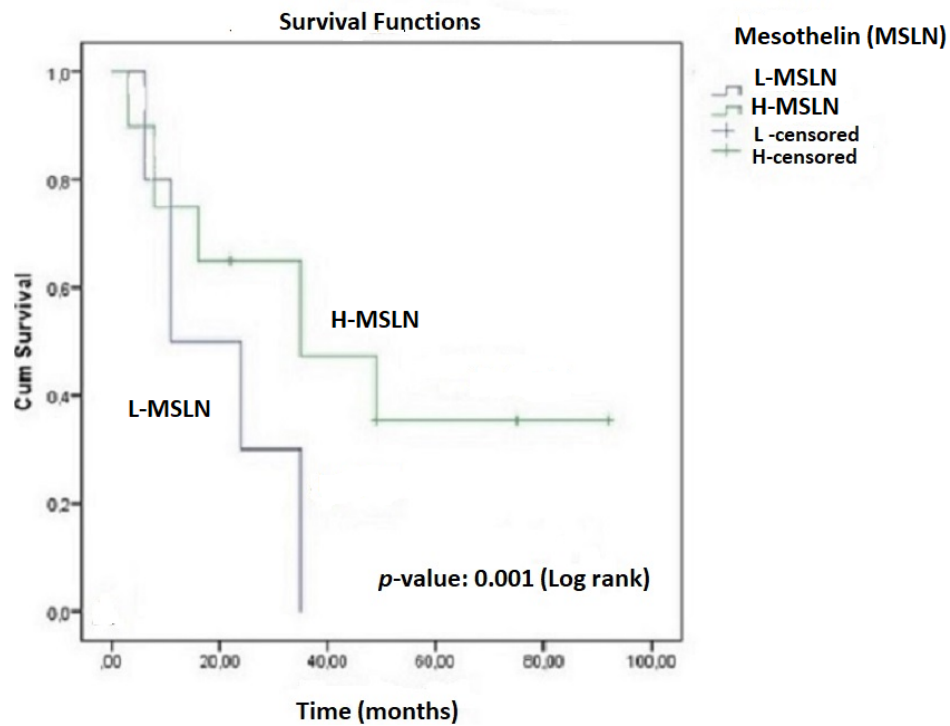

Figure 2. Kaplan-Meier survival curves stratified by mesothelin (MSLN).

Table 2. Analysis of variables affecting survival according to Kaplan-Meier analysis and Cox Proportional Multivariate Hazards Models in cases with mesothelioma.

\begin{tabular}{|c|c|c|c|c|c|}
\hline & \multicolumn{3}{|c|}{ Univariate Analysis $^{*}$} & \multicolumn{2}{|c|}{ Multivariate Analysis $^{\circ}$} \\
\hline Variables & $\mathrm{n},(*)$ & P-value & P-value & HR & $95 \%$ CI \\
\hline Age $^{1}$ & $54.5 \pm 7.28$ & 0.086 & 0.248 & 2.996 & $0.463-19.368$ \\
\hline$\leq 65 y$ & $40(33.3)$ & & & & \\
\hline$>65 y$ & $60(66.7)$ & & & & \\
\hline Gender & & 0.001 & 0.227 & 1.949 & $0.661-5.748$ \\
\hline Woman & $50(83.3)$ & & & & \\
\hline Man & $10(16.7)$ & & & & \\
\hline Mesothelioma & & 0.248 & 0.205 & 0.337 & $0.063-1.815$ \\
\hline Pl-mesothelioma & $34(56.7)$ & & & & \\
\hline Pr-mesothelioma & $26(43.3)$ & & & & \\
\hline MSLN degree & & 0.023 & 0.505 & 0.687 & $0.228-2.069$ \\
\hline H-MSLN & $20(33.3)$ & & & & \\
\hline L-MSLN & $40(66.7)$ & & & & \\
\hline TNM-stage $^{*}$ & & 0.001 & 0.044 & 3.226 & $1.032-10.082$ \\
\hline I-II & $20(33.3)$ & & & & \\
\hline III-IV & $40(66.7)$ & & & & \\
\hline $\begin{array}{l}\text { Lymph node } \\
\text { memetastase }\end{array}$ & & 0.004 & 0.631 & 0.830 & $0.389-1.772$ \\
\hline No & $32(53.3)$ & & & & \\
\hline N1-2 & $28(47.7)$ & & & & \\
\hline CT status & & 0.792 & 0.778 & 0.905 & $0.452-1.811$ \\
\hline $\mathbf{C T}(+)$ & $32(53.3)$ & & & & \\
\hline C T(-) & $28(47.7)$ & & & & \\
\hline RT status & & 0.125 & 0.246 & 2.939 & $0.475-18.20$ \\
\hline RT (+) & $14(23.3)$ & & & & \\
\hline RT (-) & $46(76.7)$ & & & & \\
\hline
\end{tabular}


While there was a significant positive relationship between the MSLN overexpression, age, and survival (Pearson's $\mathrm{r}=0.366, \mathrm{P}=0.004$, Pearson's $\mathrm{r}=0.297$, $\mathrm{P}=0.021$, respectively), a negative relationship was observed between the stage, lymph node metastasis, and MSLN overexpression (Pearson's $\mathrm{r}=-0.268, \mathrm{P}=0.038$, Pearson's $\mathrm{r}=-0.386, \mathrm{P}=0.02$, respectively). There was no correlation between other parameters (status of CT or RT, peritoneal or pleural MSM, and MSLN expression).

\section{siRNA transfection efficiency analysis}

After the siRNAs transfection of MSLN and the mRNA expression levels were analyzed with qRT-PCR. The mRNA expressions of MSLN in siRNA groups demonstrated a significant reduction according to the control group $(\mathrm{P}=0.007)$. The siRNA silencing rates were $87.5 \%$ with the genes calculation method for applied biosystems (Figure 3).

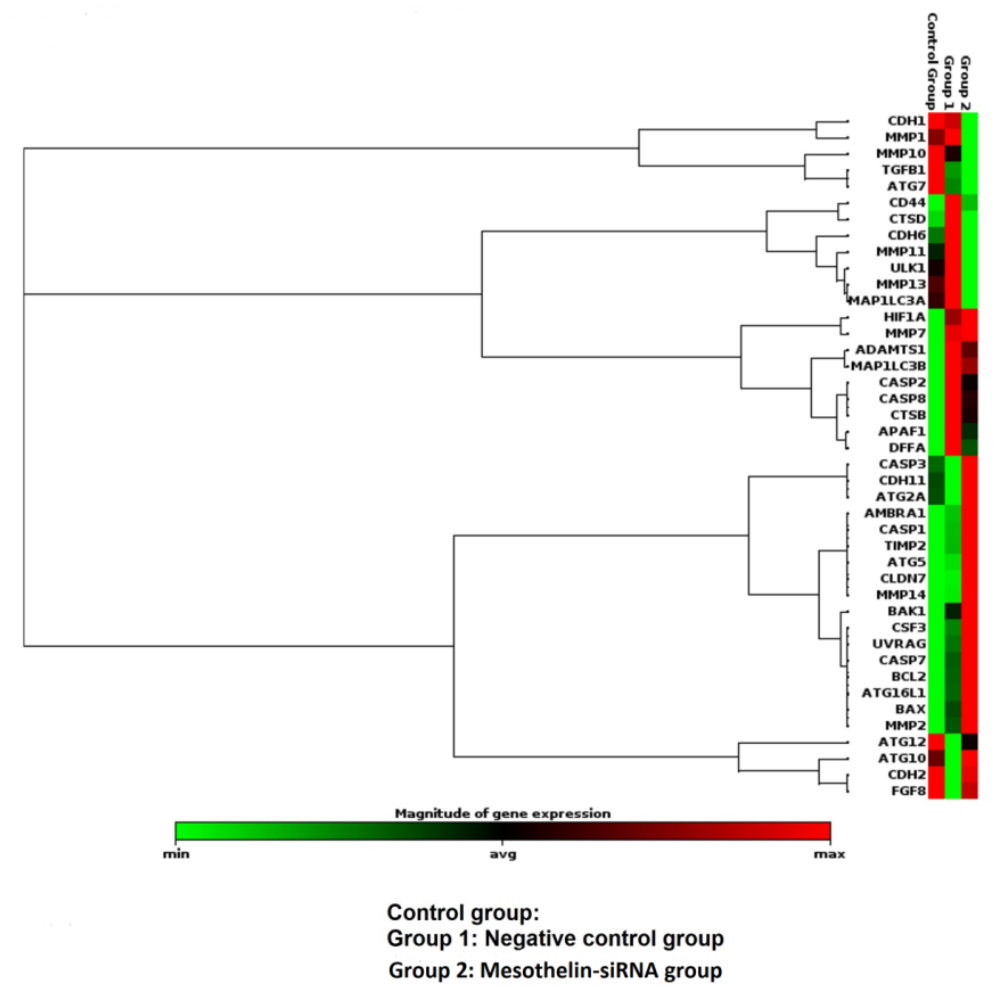

Figure 3. Heat map of the effects on gene expression data, after transfection mesothelin (MSLN) in the mesothelioma cell line. The mRNA expression obtained using qPCR for the targets selected from the genes in Table 3 is shown as a graph of the profile of the heat map. Genes clustered according to their expression patterns. The red color on the heat map indicates genes that are highly expressed compared to control; green color, genes that are expressed at alow level; whereas black color represents genes that are equal to the control.

A total of 41 genes were successfully analyzed. The MSLN silencing significantly increased apoptosis-related genes, the pro-apoptotic genes BCL2-antagonist/killer1 (BAK1), BCL2-associated X protein (BAX), and caspase1, (CASP1), and caspase7 
(CASP7). The proliferation-invasion genes were affected bidirectional by MSLN silencing. Although the proliferation-invasion-related genes, claudin7 (CLN7), colony-stimulating factor3 (CSF3), and TIMP metallopeptidase inhibitor2 (TIMP2), increased markedly, some invasion genes, cadherin 1 (CDH1) gene, matrix metallopeptidase 1 (interstitial collagenase) (MMP1), matrix metallopeptidase 10 (MMP10), matrix metallopeptidase 11 (MMP11), matrix metallopeptidase 13 (MMP13), downregulated by MSLN silencing. Autophagyrelated genes, ATG2 autophagy-related two homologs A (ATG2A), ATG16 autophagyrelated 16-like1 (ATG16L1), upregulated and UVRAG partially suppressed as shown in Table $3(\mathrm{P}<0.05)$.

Table 3. mRNA fold change and $p$-value in the mesothelioma cell line (SPC212) compared to the control group after mesothelin (MSLN) siRNA transfection.

\begin{tabular}{|c|c|c|c|c|c|}
\hline \multirow[b]{2}{*}{ Symbol } & \multirow[b]{2}{*}{ Name } & \multicolumn{2}{|c|}{ Negative Control } & \multicolumn{2}{|c|}{ MSLN siRNA } \\
\hline & & $\begin{array}{l}\text { mRNA } \\
\text { fold } \\
\text { change }\end{array}$ & P-value & $\begin{array}{l}\text { mRNA } \\
\text { fold change }\end{array}$ & P-value \\
\hline GAPDH & Glyceraldehyde-3-phosphate dehydrogenase & 1 & 0 & 1 & 0 \\
\hline APAF1 & Apoptotic peptidase activating factor 1 & 1.3755 & 0.2561 & 1.1567 & 0.58258 \\
\hline BAK1 & BCL2-antagonist/killer 1 & 1.7901 & 0.078219 & 2.8089 & 0.01962 \\
\hline BAX & BCL2-associated X protein & 1.9862 & 0.052849 & 3.7842 & 0.01128 \\
\hline BCL2 & B-cell CLL/lymphoma 2 & 1.1487 & 0.600612 & 1.4845 & 0.177637 \\
\hline CASP1 & Caspase 1, apoptosis-related cysteine peptidase & 1.6133 & 0.121328 & 5.8563 & 0.00682 \\
\hline CASP2 & Caspase 2, apoptosis-related cysteine peptidase & 1.7532 & 0.085083 & 1.3947 & 0.239448 \\
\hline CASP3 & Caspase 3, apoptosis-related cysteine peptidase & 0,7846 & 0,362011 & 1,5157 & 0,161145 \\
\hline CASP7 & Caspase 7, apoptosis-related cysteine peptidase & 1.6935 & 0.098296 & 3.2043 & 0.014948 \\
\hline CASP8 & Caspase 8 , apoptosis-related cysteine peptidase & 1.879 & 0.064756 & 1.5052 & 0.166442 \\
\hline DFFA & DNA fragmentation factor, $45 \mathrm{kDa}$, alpha polypeptide & 1.3287 & 0.303128 & 1.1096 & 0.695984 \\
\hline ADAMTS1 & ADAM metallopeptidase with thrombospondin type motif, 1 & 1.7532 & 0.085083 & 1.5157 & 0.161145 \\
\hline CD44 & CD44 molecule (Indian blood group) & 1.6702 & 0.104287 & 1.0792 & 0.777726 \\
\hline CDH1 & Cadherin 1, type 1, E-cadherin (epithelial) & 0.9075 & 0.688221 & 0.0421 & 0.005327 \\
\hline CDH11 & Cadherin 11, type 2, OB-cadherin (osteoblast) & 0.9526 & 0.830902 & 1.0867 & 0.756902 \\
\hline $\mathrm{CDH} 2$ & Cadherin 2, type 1, N-cadherin (neuronal) & 0.8409 & 0.496373 & 0,9931 & 0,961064 \\
\hline CDH6 & Cadherin 6 , type 2 , K-cadherin (fetal kidney) & 1.2397 & 0.423463 & 0.9138 & 0.707769 \\
\hline CLDN7 & Claudin 7 & 1.1728 & 0.547633 & 9.5798 & 0.004967 \\
\hline CSF3 & Colony stimulating factor 3 (granulocyte) & 2.1585 & 0.039943 & 5.8159 & 0.006861 \\
\hline CTSB & Cathepsin B & 1.434 & 0.209487 & 1.2397 & 0.423463 \\
\hline CTSD & Cathepsin D & 1.2834 & 0.358641 & 0.9794 & 0.917185 \\
\hline FGF8 & Fibroblast growth factor 8 (androgen-induced) & 0.6974 & 0.211053 & 0.9659 & 0.873735 \\
\hline HIF1A & Hypoxia inducible factor 1 , alpha subunit & 1.4641 & 0.189685 & 1.5801 & 0.133161 \\
\hline MMP1 & Matrix metallopeptidase 1 (interstitial collagenase) & 1.2226 & 0.452126 & 0.2813 & 0.016004 \\
\hline MMP2 & Matrix metallopeptidase 2 (gelatinase A) & 1.0943 & 0.736324 & 1.2834 & 0.358641 \\
\hline MMP7 & Matrix metallopeptidase 7 (matrilysin, uterine) & 1.8025 & 0.076088 & 1.8404 & 0.070127 \\
\hline MMP10 & Matrix metallopeptidase 10 (stromelysin 2$)$ & 0.5783 & 0.096759 & 0.0552 & 0.005606 \\
\hline MMP11 & Matrix metallopeptidase 11 (stromelysin 3) & 1.815 & 0.074031 & 0.3763 & 0.027224 \\
\hline MMP13 & Matrix metallopeptidase 13 (collagenase 3 ) & 1.3566 & 0.273954 & 0.3439 & 0.022584 \\
\hline MMP14 & Matrix metallopeptidase 14 (membrane-inserted) & 1.014 & 0.972742 & 1.4044 & 0.231552 \\
\hline TGFB1 & Transforming growth factor, beta 1 & 0.9013 & 0.668991 & 0.8766 & 0.595493 \\
\hline TIMP2 & TIMP metallopeptidase inhibitor 2 & 1.1487 & 0.600612 & 2.1287 & 0.041767 \\
\hline AMBRA1 & Autophagy/beclin-1 regulator 1 & 1.014 & 0.972742 & 1.1251 & 0.65684 \\
\hline ATG2A & ATG2 autophagy related 2 homolog A (S. cerevisiae) & 0.9075 & 0.688221 & 1.4044 & 0.027224 \\
\hline ATG5 & ATG5 autophagy related 5 homolog (S. cerevisiae) & 1.021 & 0.950677 & 1.366 & 0.264873 \\
\hline ATG7 & ATG7 autophagy related 7 homolog (S. cerevisiae) & 0.8526 & 0.527874 & 0.8066 & 0.411205 \\
\hline ATG10 & ATG10 autophagy related 10 homolog (S. cerevisiae) & 0.722 & 0.246871 & 1.1173 & 0.676255 \\
\hline ATG12 & ATG12 autophagy related 12 homolog (S. cerevisiae) & 0.79 & 0.37376 & 0.9013 & 0.668991 \\
\hline ATG16L1 & ATG16 autophagy related 16-like 1 (S. cerevisiae) & 1.6818 & 0.101237 & 3.2716 & 0.014381 \\
\hline MAP1LC3B & Microtubule-associated protein 1 light chain 3 beta & 1.2058 & 0.48238 & 1.1647 & 0.564917 \\
\hline ULK1 & Unc-51-like kinase 1 (C. elegans) & 1.0792 & 0.777726 & 0.9075 & 0.688221 \\
\hline UVRAG & UV radiation resistance associated gene & 1.1408 & 0.619007 & 0.3439 & 0.005606 \\
\hline
\end{tabular}




\section{siRNA-mediated MSLN silencing induced apoptosis via the caspase 3 pathway}

To determine the possible apoptotic and autophagic mechanism of MSLN contributing to SPC212 cells, we examined CASP3 and LC-3 genes expression using western blot analysis. The experiment was divided into three groups as transfection reagent (Control; C), scrambled siRNA group (Sc-siRNA), and MSLN- siRNA groups (MSLNsiRNA). Results revealed that the levels of CASP3 and LC3 genes were significantly higher in the MSLN-siRNA group. MSLN silencing was determined to significantly increase CASP3, but not change LC3 $(\mathrm{P}<0.001$ and $\mathrm{P}=0.24)$ as shown in Figure 4. These results suggested that the inhibition of MSLN could induce the apoptosis of SPC212 cells via the CASP3 pathway.
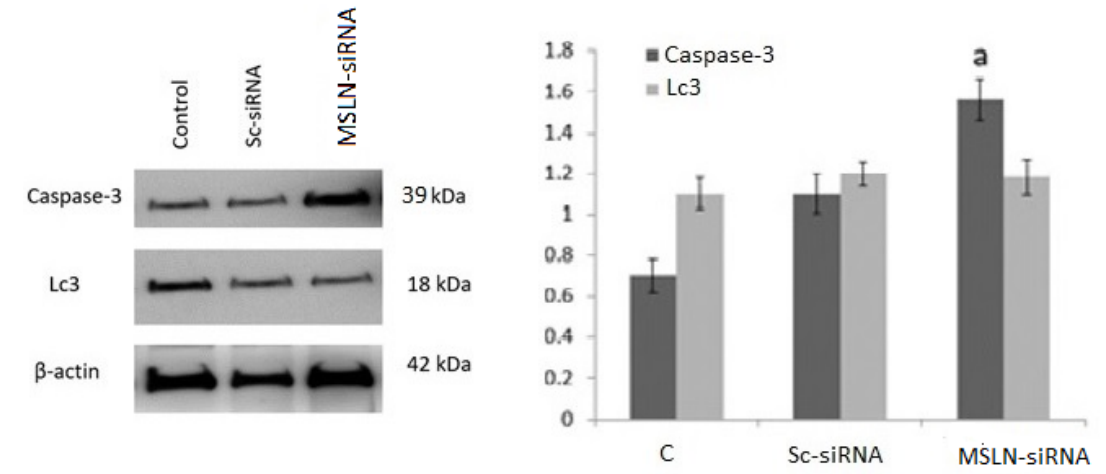

Figure 4. Western blot analysis of caspase 3 and LC-3 proteins in SPC212 cells. The $\beta$-actin was used as an internal control. SPC212 cells were treated with Sc-siRNA and mesothelin (MSLN)-siRNA for 48 hours. The densitometer-intensity data of the protein bands of each blot is represented as mean \pm SEM from three independent experiments. a indicates $\mathrm{p}<0.05$ versus control as measured by a one-way ANOVA test.

\section{DISCUSSION}

Mesothelioma is fatal cancer where survival could be up to a year with current treatment modalities. Naturally, we need to understand the etiopathogenesis of disease better for more favorable treatment outcomes (NCCN, 2021; Kim et al., 2006). MSLN, a glycosylphosphatidylinositol-anchored membrane glycoprotein, is overexpressed in some epithelial malignancies (Chang et al., 2021).

Although their biological functions are still not fully understood, they have effects in the activation and regulation of matrix metalloproteinases, which are important in cellular invasion and metastasis, and Nf-kB pathway, which is an autocrine growth factor. MSLN expressions have been shown to vary with the activation of Wnt-1 proteins secreting glycoproteins through intracellular pathways in the process of cell growth, differentiation, apoptosis, and high expression rates were associated with poor prognosis in some cancers (Chang et al., 2009; Chang et al., 2011). Therefore, it has been considered an excellent target for targeted therapy. The relationship between MSLN expression and survival is contradictory in the literature (Roe et al., 2008; Feng et al., 2020). H-MSLN was related to improved survival in the present study. Similar to our study, Roe et al. showed that 
overexpression of MSLN was a favorable factor for survival, but Feng F. et al. demonstrated that longer survival was in mesothelioma cases with lower MSLN expression (Roe et al., 2008; Feng et al., 2020). The MSLN expression rates (66\%) in our study were also compatible with the literature (Inaguma et al., 2017).

In recent studies, a correlation has been found between serum MSLN levels and anti-MSLN treatments (Katz et al. 2021). Similarly, some researchers have shown that MSLN overexpression causes cell growth and metastasis in NCH-I, Mero-14, and IstMes 2 mesothelioma cell lines, and the combination of MSLN-siRNA and cytotoxic CT suppresses invasion, induces apoptosis (Melaiu et al., 2014). Researchers have investigated MSLN-targeted therapies such as recombinant immunotoxin (SS1P and LMB-100)or adoptive T-cell immunotherapies, antibody drug-drug conjugates (anetumab ravtansine, BMS-986148, and BAY2287411), chimeric monoclonal antibody (amatuximab) with this logic in the treatment of mesothelioma (Ho et al., 2005; Hassan et al., 2013; Yeo et al., 2021). The results from these studies are very encouraging, but anti-MSLN therapies are not currently suitable for clinical use.

Apart from mesothelioma, it has been shown that MSLN can be pharmacologically inhibited in cancers with MSLN overexpression such as pancreas cancer (Melaiu et al., 2014). In recent years, small interfering RNAs (siRNAs) that stop gene expression and impede the development and progression of several cancers became famous in cancer treatment. siRNAs serve as an RNA guide for proper microRNA (miRNA) degradation. It was demonstrated to have a prognostic function in certain cancers (Lai et al., 2016; Kaur et al. 2018). We identified in which stage MSLN should be placed in carcinogenesis and investigated in vitro whether silencing of these elements with siRNAs blocked the carcinogenesis stages. To date, our study is the first to demonstrate the silencing function of MSLN with siRNAs in the SPC212 cell line. We also evaluated in vivo how the MSLN expression rates in mesothelioma case specimens were reflected on survival.

The stability between cell proliferation and dying cells is often disrupted. In the stages of carcinogenesis, there will be problems in the perception of the signals that will put the cells on the apoptotic pathway

Although Ombretto et al. showed that MSLN silencing did not affect apoptosisrelated genes, CASP3 and 7, we showed that apoptosis-related genes such as CASP1-7 and the pro-apoptotic genes, BAK1, BAXs, were partially activated in this study (Melaiu et al., 2014). As is well known, epithelial-mesenchyme transition (EMT) is a significant barrier to the metastasis of cancer cells. Metastases occur when no barrier is present. In the present, proliferation-invasion-related genes such as $\mathrm{CDH} 1$, an adhesion molecule in epithelial cells, and MMP10, 11, and 13 were downregulated by MSLN silencing as a defense mechanism against the stages of cell carcinogenesis. There is data showing that MMP7 has a function in etiopathogenesis via MAPK/ERK and JNK pathways. MMP7, via increasing IL6 levels, induces the invasion of mesothelioma and leading to apoptosis resistance via TNF alpha in the carcinogenesis of mesothelioma (Chang et al., 2012). Correlation in the MMP7 and mesothelioma are contradictory in the literature (Ali et al., 2013). In the present study, we didn't detect any correlation between the silencing of MSLN with siRNA and the MMP7. As noted by Chang et al. (2012) it was conceived that different types of cancer could spread through different pathways. We thought that MMP7 was not used in the proliferation pathways of mesothelioma. 
Epithelial cancers could invade through cell adhesion gene suppression via EMT loss. In certain tumors, the invasion could occur via E cadherin expression. Shamir et al. had shown that epithelial cancer can metastasize through the loss of epithelial cell-cell junctions and epithelial-mesenchymal transition by suppressing cell adhesion genes, or in some tumors, invasion can occur via the induction of Twist1 expression without the loss of E cadherin expression. The results of our study revealed that SPC212 cells proliferate via down-regulated CDH1 protein by siRNA-mediated MSLN. We thought that the efficacy of $\mathrm{CDH} 1$ protein in the invasion mechanism of mesothelioma was related to the MSLN and that invasion in mesothelioma occurs predominantly in the loss of CDH1 (Shamir et al., 2014). Similar to our results, Wang et al., also showed in their studies that gene-specific MSLN silencing reduced the invasion capacity of cancer cells. (Wang et al., 2012).

Another gene that plays an important role in EMT regulation is CLND7. Studies demonstrated that when CLDN7 was disturbed by a defect or disorder, EMT structure could break down, leading to the spread of a malignant event (Yilmaz et al, 2009). In the current study, it was observed that MSLN silencing upregulated the CLND7 protein. This finding highlights the critical role of CLND7 in the mesothelioma proliferation via MSLN.

CSF3 suppresses $\mathrm{T}$ cell functions and count around the tumor microenvironment due to higher radical oxidative stress (ROS) levels (Vassilev et al., 2015). In the present study, we observed that MSLN silencing could increase CSF3. Also, we suggested that this might contribute positively to cytotoxic T cell functions indirectly. The upregulated CLND7 and CSF3 proteins have been protective to invasion in the SPC212 cells.

Fibroblast growth factor 8 (FGF-8b) and VEGF serve as angiogenic growth factors in endothelial cell growth and tube formation in tumorigenesis. MSLN silencing did not affect the angiogenesis pathway in the present study (Ren et al., 2016). We conclude that MSLN does not function through angiogenesis.

Hypoxia-inducible factor 1a (HIF-1a) is a molecule that plays a role in cellular proliferation and invasion mechanisms. This is done via the destruction of the EMT zone through metalloproteinases released by the tumor cells. In a study by Zhang et colleagues on ovarian cancer cell lines, it was reported that siRNAs could reduce HIF-1a expression via MMP13 inhibition. In our study, we demonstrated that MMP 10, 11, 13 could downregulate via MSLN silencing independent from the HIF-1a in our study similar to Zhang et al.'s study (Zhang et al., 2019).

TIMP2 silencing by miR-205-5ps acts together with MMP2 in the activation of pro-MMP2 to prevent cancer cell progression and migration (Nagai et al., 2018). In the present study, the TIMP2 was upregulated by MSLN silencing in mesothelioma cell lines. It was appeared to predominantly induce proliferation of mesothelioma as the MSLN silencing effect in the SPC212 cells.

There is a shortage or lack of transforming growth factor- $\beta$ I (TGF $\beta I$ ) in the etiopathogenesis of several cancers (Wen et al., 2011). TGF $\beta$ I exhibits a tumor-suppressing effect via the PI3K/Akt/mTOR pathway. While TGF $\beta$ I acts like TSG in early-stage tumor growth, it helps the breakdown of EMT and invasion of malignant cells in advanced-stage tumors. Dong et al. demonstrated that the silencing maternal gene 3 expression (MEG3) with siRNA suppressed the proliferation of hepatocellular cancer due to TGF $\beta$ I inhibition (Dong et al., 2019). In the current study, it was observed that TGF $\beta 1$ couldn't be silenced by siRNA-mediated MSLN. In conclusion, we concluded that the TGF $\beta 1$ gene does not play a role through MSLN in mesothelioma proliferation. 
Autophagy has a bilateral function in cancer invasion. The early stages of cancer invasion prevent distant metastasis in the limited stage by preventing necrosis and inflammation. The advanced cancer stage helps the tumor cells breach the EMT zone and adhere-reside or stay dormant in the new microenvironment. There is a strong relationship between the apoptotic and autophagy pathways. This relationship mostly occurs via the genes that provide mitochondrial regulation of apoptosis, affecting the autophagy pathway. Certain pro-apoptotic signals also induce autophagy. Although the relationship between autophagy and miRNAs is not known entirely, there are BECN1-dependent and independent autophagy types (Towers et al., 2020). The current study observed that autophagy could be partially induced by increasing the ATG16L1 and the ATG2A, which has a function in the early stages of vesicle formation.

The present study has several limitations. First, the number of cases diagnosed with mesothelioma was limited. Second, we did not investigate other probable interweaving modifications of these genes and changes in genetic variations that could emerge along the SPC212 cell line. Third, only one mesothelioma cell line was used. Forth, examination of MSLN only was used immunohistochemistry, not blood samples.

In conclusion, it was determined that histopathological samples obtained from mesothelioma cases were MSLN immune reactive. We thought that MSLN overexpression played an important role in the mesothelioma proliferation mechanism, in early-stage, and without lymph node involvement, especially. In addition, our data showed that higher MSLN is a favorable factor in terms of survival. Immunohistochemically, low MSLN expression did not provide a survival advantage in mesothelioma patients in vivo. siRNAmediated MSLN silencing partially contributed to the proliferation of SPC212 cells, in vitro. While the target of MSLM silencing in early-stage mesothelioma can slow the progression of cancer, the methods of MSLM silencing may cause progression in cases with advanced-stage mesothelioma. The potential role of MSLN in the MSM cells is quite complicated. MSLN-targeted therapies in MSM treatment can be planned carefully according to the stage. siRNA-mediated MSLN silencing may be an alternative in the early stage of mesothelioma treatment. The studies that can increase the functionality of MSLN may also be among new targeted therapies. As a result, further studies should be carried out to encourage the development of new therapeutic targets.

\section{ACKNOWLEDGMENTS}

The authors are very thankful to the referee for improving the paper.

\section{ETHICS APPROVAL}

This study was approved by the Ethical Committee at the University of Firat, Medical Faculty, (11.10.2016, 17/03).

\section{FUNDING}

The authors declared that this study has received financial support from the Scientific Research Project of Firat University (no: TF.15.47/ 2015). 


\title{
CONFLICTS OF INTEREST
}

\author{
The authors declare no conflict of interest.
}

\section{REFERENCES}

Alì G, Borrelli N, Riccardo G, Proietti A, et al. (2013). Differential expression of extracellular matrix constituents and cell adhesion molecules between malignant pleural mesothelioma and mesothelial hyperplasia. J. Thorac. Oncol. 8: 1389-1395

Baas P, Scherpereel A, Nowak AK, et al. (2021). First-line nivolumab plus ipilimumab in unresectable malignant pleural mesothelioma (CheckMate 743): A multicentre, randomized, open-label, phase 3 trial. Lancet. 397: 375-386.

Bharadwaj U, Marin-Muller C, Li M, Chen C, et al. (2011). Mesothelin overexpression promotes autocrine IL-6/sIL-6R trans-signaling to stimulate pancreatic cancer cell proliferation. Carcinogenesis. 32: 1013-1024.

Bharadwaj U, Marin-Muller C, Li M, Chen C, et al. (2011). Mesothelin confers pancreatic cancer cell resistance to TNFalpha-induced apoptosis through Akt/PI3K/NF-kappaB activation and IL-6/Mcl-1 overexpression. Mol. Cancer. 10: 106.

Carter JH, Douglass LE, Deddens JA, et al. (2004). Pak-1 expression increases with the progression of colorectal carcinomas to metastasis. Clin.Cancer Res. 10: 3448-3456.

Chang MC, Chen CA, Chen PJ, Chiang YC, et al. (2012). Mesothelin Enhances Invasion of Ovarian Cancer by Inducing MMP-7 through MAPK/ ERK and JNK Pathways. Biochem. J. 442: 293-302.

Chang MC, Chen CA, Hsieh CY, Lee CN, et al. (2009). Mesothelin inhibits paclitaxel-induced apoptosis through the PI3K pathway. Biochem. J. 424: 449-458.

Chang K and Pastan I (1996). Molecular cloning of mesothelin, a differentiation antigen present on mesothelium, mesotheliomas, and ovarian cancers. Proc. Natl. Acad. Sci. 93: 136-140.

Del Bano J, Florès-Florès R, Josselin E, Goubard A, et al. (2019). A Bispecific Antibody-Based Approach for Targeting Mesothelin in Triple-Negative Breast Cancer. Front Immunol. 10: 1593.

Dong H, Zhang Y, Xu Y, Ma R, et al. (2019). Downregulation of long non-coding RNA MEG3 promotes proliferation, migration, and invasion of human hepatocellular carcinoma cells by upregulating TGF- $\beta 1$. Acta Biochim. Biophys. Sin. 51: 645-652.

Edge SB (2017). American Joint Committee on Cancer. AJCC cancer staging manual. 8th eds. Springer, New York.

Feng F, Zhang H, Zhang Y and Wang H (2020). Level of mesothelin expression can indicate the prognosis of malignant pleural mesothelioma. Transl. Cancer Res. 9: 7479-7485.

Fukumoto I, Hanazawa T, Kinoshita T, Kikkawa N, et al. (2015). MicroRNA expression signature of oral squamous cell carcinoma: Functional role of microRNA-26a/bin the modulation of novel cancer pathways. Br. J. Cancer. 112: 891-900.

Gozuacik D and Kimchi A (2004). Autophagy as a cell death and tumor suppressor mechanism. Oncogene. 23: 28912806.

Hassan R, Miller AC, Sharon E, Thomas A, et al. (2013). Major cancer regressions in mesothelioma after treatment with an anti-mesothelin immunotoxin and immune suppression. Sci. Transl. Med. 5: $208 \mathrm{ra} 147$.

Ho M, Hassan R, Zhang J, Wang QC, et al. (2005). Humoral immune response to mesothelin in mesothelioma and ovarian cancer patients. Clin. Cancer Res. 11: 3814-3820.

Inaguma S, Wang Z, Lasota J, Onda M, et al. (2017). Comprehensive immunohistochemical study of mesothelin (MSLN) using different monoclonal antibodies 5B2 and MN-1 in 1562 tumors with evaluation of its prognostic value in malignant pleural mesothelioma. Oncotarget. 8: 26744-26754.

Katz SI, Roshkovan L, Berger I, Friedberg JS, et al. (2021). Serum soluble mesothelin-related protein (SMRP) and fibulin-3 levels correlate with baseline malignant pleural mesothelioma (MPM) tumor volumes but are not useful as biomarkers of response in an immunotherapy trial. Lung Cancer. 154: 5-12.

Kaur K, Rath G, Chandra S, Singh R, et al. (2018). Chemotherapy with si-RNA and Anti-Cancer Drugs. Curr. Drug Deliv. 15: 300-311.

Kim R, Emi M and Tanabe K (2006). Cancer immunosuppression and autoimmune disease: beyond immunosuppressive networks for tumor immunity. Immunology. 19: 254-264.

Lai XJ, Cheng XY and Hu LD (2016). microRNA 421 induces apoptosis of c-33a cervical cancer cells via downregulation of Bcl-xL. Genet. Mol. Res. 15: gmr15048853.

Melaiu O, Stebbing J, Lombardo Y, Bracci E, et al. (2014). MSLN Gene silencing has an anti-malignant effect on cell lines overexpressing mesothelin deriving from malignant pleural mesothelioma. PLoS One. 21: 9:e85935.

National Comprehensive Cancer Network (NCCN) Clinical Practice Guidelines in Oncology. Version 2. 2021. Malignant Pleural Mesothelioma. www.nccn.org/professionals/physician_gls/pdf/mpm.pdf (2021, accessed 16 February 2021) 
Nagai H, Hasegawa S, Uchida F, Terabe T, et al. (2018). MicroRNA-205-5p suppresses the invasiveness of oral squamous cell carcinoma by inhibition TIMP 2 expression. Int. J. Oncol. 52: 841-850.

Ren C, Chen H, Han C, Fu D, et al. (2016). The anti-apoptotic and prognostic value of fibroblast growth factor 9 in gastric cancer. Oncotarget. 7: 36655-36665.

Roe OD, Creaney J, Lundgren S, Larsson E, et al. (2008). Mesothelin-related predictive and prognostic factors in malignant mesothelioma: a nested case-control study. Lung Cancer. 61: 235-243.

Rump A, Morikawa Y, Tanaka M, Minami S, et al. (2004). Binding of ovarian cancer antigen CA125/MUC16 to mesothelin mediates cell adhesion. J. Biol. Chem. 279: 9190-9198.

Shamir ER, Pappalardo E, Jorgens DM, et al. (2014). Twist1-induced dissemination preserves epithelial identity and requires E-cadherin. J. Cell. Biol. 204: 839-856.

Srivastava A (2015). A novel link between FMR gene and the JNK pathway provides clues to possible role in malignant pleural mesothelioma. FEBS Open Bio. 5: 705- 711.

Towers CG, Wodetzki D and Thorburn A (2020). Autophagy-dependent cancer cells circumvent loss of the upstream regulator RB1CC1/FIP200 and loss of LC3 conjugation by similar mechanisms. Autophagy. 16: 1332-1340.

Van Zandwijk N, Reid G and Baas P (2020). Editorial: Emerging Therapies for Malignant Mesothelioma. Front Oncol. 10

Vassilev L, Ranki T, Joensuu T, Jäger E, et al. ( 2015). Repeated intratumoral administration of ONCOS-102 leads to systemic antitumor CD8 T-cell response and robust cellular and transcriptional immune activation at tumor site in a patient with ovarian cancer. Oncoimmunology. 4: e1017702.

Wang K., Bodempudi V, Liu Z, et al. (2012). Inhibition of mesothelin as a novel strategy for targeting cancer cells. PloS one. 7: $\mathrm{e} 33214$.

Wen G, Hong M, Li B, LIao W, et al. (2011). Transforming growth factor- $\beta$-induced protein (TGFBI) suppresses mesothelioma progression through the Akt/mTOR pathway. Int. J. Oncol. 39: 1001-1009.

Yilmaz M and Christofori G. (2009). EMT, the cytoskeleton, and cancer cell invasion. Cancer Metastasis Rev. 28: 1533.

Yalcin E, Pala S, Atılgan R, et al. (2019). Is there any difference between endometrial hyperplasia and endometrial carcinoma in terms of expression of TRPM2 and TRPM7 ion channels? Turk. J. Med. Sci. 49: 653-660.

Yeo D, Castelletti L, van Zandwijk N, et al. (2021). Hitting the Bull's-Eye: Mesothelin's Role as a Biomarker and Therapeutic Target for Malignant Pleural Mesothelioma. Cancers. 13: 3932.

Zhang H, Yang Q, Lian X, Jiang P, et al. (2019). Hypoxia-Inducible Factor-1 $\alpha$ (HIF-1 $\alpha$ ) Promotes Hypoxia-Induced Invasion and Metastasis in Ovarian Cancer by Targeting Matrix Metallopeptidase 13 (MMP13). Med. Sci. Monit. 25: $7202-7208$ 